\title{
Artificial Intelligence in Public Health Dentistry
}

\section{SAHRISH TARIQ*1, NIDHI GUPTA² (D), PREETY GUPTAiD, ADITI SHARMA4 (iD)}

The educational needs must drive the development of the appropriate technology. They should not be viewed as toys for enthusiasts. Nevertheless, the human element must never be dismissed. Scientific research will continue to offer exciting technologies and effective treatments. For the profession and the patients, it serves to benefit fully from modern science, new knowledge and technologies must be incorporated into the mainstream of dental education. The technologies of modern science have astonished and intrigued our imagination. Correct diagnosis is the key to a successful clinical practice. In this regard, adequately trained neural networks can be a boon to diagnosticians, especially in conditions having multifactorial etiology.

KEYWORDS: Artificial Intelligence, Database, Machine Learning, Surveillance

\section{INTRODUCTION}

"Our intelligence is what makes us human, and AI is an extension of that quality"

Yann le Cun

Humankind has given itself the scientific epithet homo sapiens - man the astute, due to the fact our intellectual capacities are so indispensable to our existence and our sense of self. A human brain is an inimitable form composed of networks of interlinked neurons which transmit signals throughout the body. This unexampled nature of the human brain has usually made researchers and scientists inquisitive from time immemorial.

History dates back to as early as four hundred BC when Plato envisaged a basic model of brain function. Since then, the field of technology has witnessed numerous innovations that may simulate the functioning of the human intellect. The need for the constant quest has given upward thrust to what's referred to as artificial intelligence (AI), which is an exceedingly advanced system to mimic the functioning of the human brain. ${ }^{1}$

Artificial intelligence (AI) is defined as: 'a field of science and engineering concerned with the computational understanding of what is commonly called intelligent behaviour, and with the creation of artifacts that exhibit such behaviour'.
The term artificial intelligence was first coined by John McCarthy in 1956. The programs of artificial intelligence technology like expert structures, game playing, and theorem-proving, natural language processing, image reputation and robotics in numerous fields like telecommunication and aerospace have grown manifold.

Technology has additionally revolutionized the sector of medicine and dentistry in the last decade, ranging from online scheduling of appointments, online check-ins in medical centres, digitization of medical records, reminder calls for follow-up appointments and immunization dates for children and pregnant females to drug dosage algorithms and adverse effect warnings while prescribing multidrug combinations and much more.

\section{AI IN MEDICINE}

The growing use of information systems in healthcare and the sizable boom of clinical databases require conventional facts analyses to be adjusted to a new computational model. Methods primarily based on artificial intelligence algorithms have found innumerable implementations in biomedical sciences. AI in standard and in medicine or dentistry, in particular, began outgaining its foothold with the arrival of data computing in addition to cloud computing potential and availability of the full-size quantity of data collection. AI has now been proven

(C) Sahrish Tariq et al. This is an open access article distributed under the terms of the Creative Commons Attribution License CC-BY-NC 4.o, which permits unrestricted use, distribution and reproduction in any medium, provided the use is not commercial and the original author(s) and source are cited. Submitted on: 16-Nov-2021; Accepted on: 26-Dec-2021 
to be a powerful tool in establishing the correct diagnosis of numerous medical conditions, and in a few instances, more efficient. ${ }^{2,3}$

Unlike humans, AI never wants to sleep. Machine learning models could be used to observe the vital signs of patients receiving essential care and alert clinicians if certain risk factors increase. Artificial intelligence is also being applied to surgical robotics as it will determine patterns inside surgical procedures to improve best practices and to improve a surgical robots' control accuracy to submillimetre precision. Robots have a superhuman ability to repeat actual motions. This is extremely helpful in hair transplant surgeries. Surgeons are able to move the camera just by moving their eyes. Surgeons are using robotic surgery platforms that use microinstrumentation, flexible robotic procedures. Robotics improve outcomes for patients by accessing and treating disease through the body's natural openings. The platforms integrate endoscopes, instruments, and navigation into a single platform, allowing physicians to better conduct needs interventions. $^{2}$

\section{APPLICATION OF ARTIFICIAL INTELLIGENCE IN DENTISTRY}

In the field of dentistry, AI is steadily making inroads into the field of radiography, with a focus on diagnostic records of virtual IOPAs/RVGs, 3-D scans, and cone-beam computed tomography. AI-driven custom-designed orthodontic treatments are a recent innovation. The use of computer-aided designs and computer-aided production technology for a precision fit of the prosthesis is another step forward in the field of restorative and prosthetic may also have a significant impact on orofacial and craniofacial prosthetics. ${ }^{5}$ Thus, AI plays a critical role in every sector, from endodontics for apex location dentistry. ${ }^{4}$ This will not only help dentistry, but also in implantology for making precise surgical guides and determining cortical bone thickness.

\section{ROLE OF AI IN PUBLIC HEALTH}

Compared to humans, artificial intelligence-based virtual dental assistants can perform several tasks with greater precision, fewer errors, and less manpower. Some of these tasks include, booking and coordinating regular appointments according to the ease of the patients and dentists, alerting the patients and dentists about check-ups, each time any genetic or lifestyle records suggests expanded susceptibility to dental diseases, assisting the clinical diagnosis and treatment planning (figure 1). AI additionally promises to make healthcare more participatory, specifically if patients offer their data actively. Patients can be empowered through self-monitoring and self-management. Using these constantly collected data can also help to overcome the disadvantages of on-off-medicine, wherein patients are seen only for a few minutes, even as maximum health conditions are generally acquired over years, and come and go in intervals (e.g., periodontal disease). Continuous non-invasive monitoring of health and behaviour will enable a much deeper, individual understanding of the drivers and processes underlying health and disease. ${ }^{6}$ Diagnostic and treatment costs can be decreased, thereby relieving healthcare systems burdened through an ageing society with an increasing number of complex, chronically ill cases. AI can also assist to address shortages in workforce, as observed and

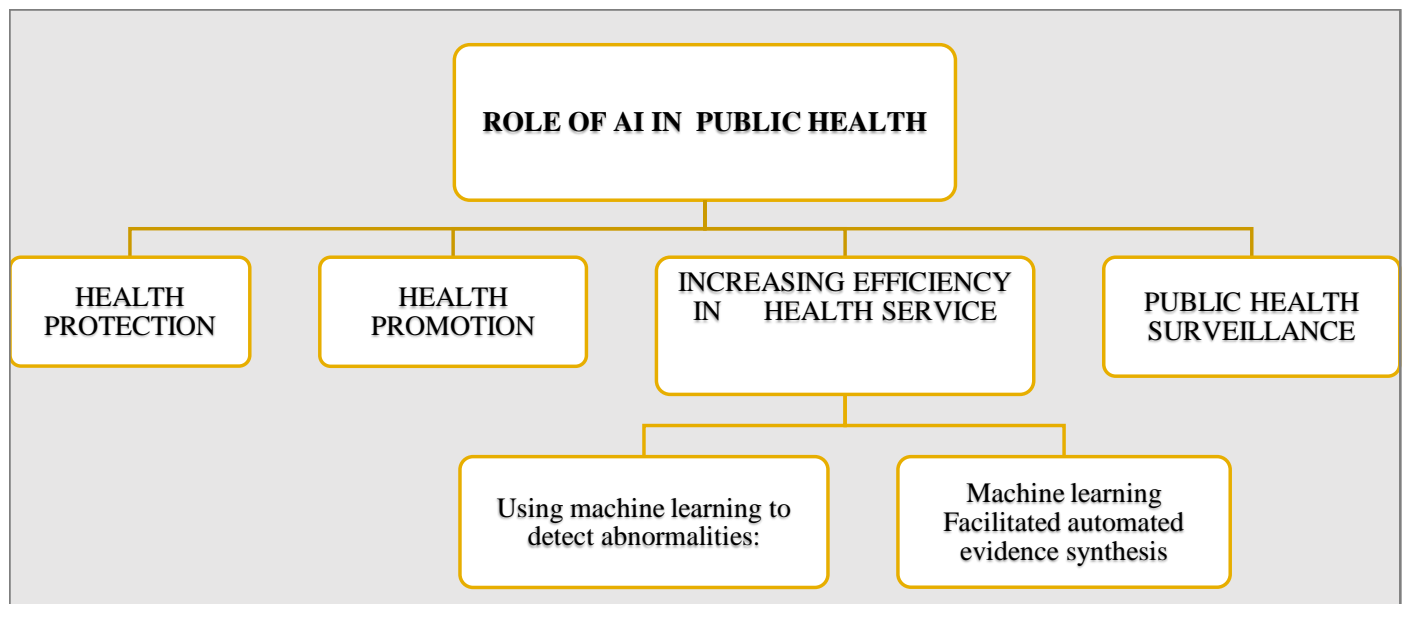

Figure 1. Role of Artificial Intelligence in Public Health 
expected to continue in many parts of the globe, thereby assisting to attain the World Health Organization (WHO) Sustainable Development Goals.

Artificial Intelligence has been implemented in academic research and in inference tasks throughout the broader economic system with demonstrable success, however much less so for the core functions of public health, specifically protecting and promoting the health of populations. To date, vision statements at the future of public health have centred at the technical possibilities of artificial intelligence and much less on how social determinants would possibly have an impact on outcomes achieved with the aid of using it. Artificial intelligence has the capability to enhance the performance and effectiveness of processes throughout an expanded public health system.

\section{HEALTH PROTECTION}

The potential use of artificial intelligence in health promotion is by analysing patterns of data for almost real-time surveillance and disease detection. For example, it may be used to screen and identify suspected changed mucosa undergoing premalignant and malignant alterations, and it can be used to diagnose and treat oral cavity lesions. Even minute changes in single pixels that would otherwise go unnoticed by the naked eye are recognised. It may also provide emergency teleassistance in cases of dental emergencies when the dental health care expert cannot be contacted.

\section{HEALTH PROMOTION}

AI offers targeted and personalised health advice based on risk profile and behavioural pattern. The skills of individual experts vary based on their prior knowledge and work experience. This is one of the reasons that people's interest in implementing computer-aided decision making has increased. For example, alerting the dental healthcare provider about any relevant medical history, that might accurately predict a genetic predisposition for oral diseases for a large population.

\section{INCREASING EFFICACY OF HEALTH SERVICES}

a). Using machine learning to detect abnormalities: Clinical Decision Support System, presents information to medical personals, patients or populations to provide efficacious and better health outcome for both individuals and populations health outcome for both individuals and populations in general. AI is already playing a prominent role in medical imaging. AI powered by artificial neural networks can be just as effective as human radiologists at detecting signs of oral cancer as well as other conditions. In addition it might help clinicians spot early signs of disease.

b). Machine learning-facilitated automated evidence synthesis: The most tedious part is to enter structured information into the system, but with the arrival of voice recognition and the potential of artificial intelligence program to identify and extract information from scanned paperwork, this procedure has been simplified. Added to that is the interactive interphase which is designed to help the health care expert to comprehend a vast amount of information more efficaciously than human assistants and concurrently bridge the distance between the doctor and the affected person.

The important targets consist of documentation and scientific coding, organizing scientific complexity, storing and retaining patients' databases, monitoring patients' orders, monitoring health situations in addition to preventive measures, such as setting up regular reminders for patients who are on tobacco or smoking cessation programs.

\section{PUBLIC HEALTH SURVEILLANCE}

Public health surveillance is the process of detecting, characterizing, tracking, and responding to disease outbreaks, other health threats (such as bioterrorist attacks, radiation exposure, or contamination of food or water supply), and other population-related health patterns (such as periodontal disease, oral cancer, or malnutrition). ${ }^{7}$ These surveillances occur at local, state, national, and global levels, and often require the integration of multiple organizations (e.g., hospitals, pharmacies, and public, state, and political health organizations) to achieve timely, focused, and effective response to emerging health events.

In this work, we focus on the role that artificial intelligence and mechanical learning can play in assisting public health with early, automatic detection of emerging outbreaks and other healthrelated patterns. Over the past decade there have been significant improvements in outbreak detection methods, including (but not limited to) local and temporary data analysis, integration of 
multiple data streams, and improved detection performance measurement metrics. ${ }^{8}$

While many existing monitoring systems rely heavily on basic statistical methods such as time series analysis and the knowledge of public health professionals, we believe that the field of diagnostics is undergoing a major paradigm shift due to the huge increase in number, quantity, and complexity of available data sources. Current diagnostic programs rely heavily on large data from non-traditional sources, from internet search queries and usergenerated web content, to electronic health records, to continuous data streams from sensory networks, mobile phones, and other location-aware devices. This shift in data analysis at the public scale will require a consistent shift in methods used in functional diagnostic systems, including techniques from artificial intelligence, machine learning, and data mining to determine large amounts of data, appropriate patterns, and assist in public health decision making. Experts will rely heavily on tools and systems that use advanced statistical methods to accurately distinguish associated ineffective patterns, scalable algorithms to process large amounts of complex, highly-dimensional data, and machine learning methods to further improve system performance from user feedback.

\section{CONCLUSION}

The zenith of artificial intelligence along with digitization has seen a new era withinside the subject of dentistry and its future aspects seem extraordinarily promising. Thus, one motive to study is to learn more about ourselves. Another motive is to construct clever entities in addition to recognize them. Artificial intelligence nonetheless is in its infancy and in no manner can supersede human intelligence and skill. Nevertheless, this technology is assisting in creating more awareness concerning oral and maxillofacial disease, risk factors and at the same time encouraging patients for early treatment. AI has produced much large and staggering merchandise even at this early level. It's far clear that computer systems with human-level intelligence (or higher) might have an enormous impact on our lives and on the course of civilization ahead.

While, in no ways, there exists a doubt withinside the supremacy of integrating AI into practice, it in no way substitutes the role of a dentist, since clinical practice is not only about diagnoses but additionally also includes correlating with clinical findings and imparting personalized patient care. Although AI can help in several ways, the very last call needs to be made by a dentist as dentistry is a multidisciplinary approach.

Predicting The Future Isn't Magic, Its AI

\section{REFERENCES}

- Davis Waters

1. Turing AM. Computing machinery and intelligence. Mind 1950;59:433-6o.

2. Ramesh AN, Kambhampati C, Monson JRT, Drew PJ. Artificial intelligence in medicine. Annals of the Royal College of Surgeons of England 2004;86(5): 334-8. https://doi.org/10.1308/147870804290 3. Deshmukh SV. Artificial intelligence in dentistry. J Int Clin Dent Res Organ. 2018;10:47-8. https://doi.org/10.4103/jicdro.jicdro_17_18

4. Buchanan JA. Experience with virtual reality-based technology in teaching restorative dental procedures. J Dent Educ. 2004;68(12):1258-65.

5. Kikuchi H, IkedaM, Araki K, Evaluation of a Virtual Reality Simulation System for Porcelain Fused to Metal Crown Preparation at Tokyo Medical and Dental University. Journal of Dental Education 2013;77:782-92.

6. Joda T. Health Data in Dentistry: An Attempt to Master the Digital Challenge. Public Health Genomics 2019;22:1-7. https://doi.org/10.1159/000501643

7. Cooper GF, Dash D, Levander J, Wong W-K, Hogan W, Wagner M. Bayesian Biosurveillance of Disease Outbreaks. Proc. $20^{\text {th }}$ Conf. Uncertainty in Artificial Intelligence;2004,94-103.

8. Kulldorff, M, Mostashari F, Duczmal L, Katherine Yih W, Kleinman K, Platt R. Multivariate scan statistics for disease surveillance. Statist Med. 2007;26:1824-33. https://doi.org/10.1002/sim.2818. 
Cite this article as:

Source of support: Nil, Conflict of interest: None declared

Tariq S, Gupta N, Gupta P, Sharma A. Artificial Intelligence in Public Health Dentistry. Int Healthc Res J. 2021;5(9):RV1-RV5. https://doi.org/10.26440/IHRJ/0509.12489

\section{AUTHOR AFFILIATIONS: ${ }^{*}$ Corresponding Author)}

1. Post Graduate Student

2. Professor \& Head (https://orcid.org/oooo-0oo2-4735-9462)

3. Reader (https://orcid.org/oooo-0002-5382-3372)

4. Senior Lecturer (https://orcid.org/oooo-ooo1-8818-493X)

Department of Public Health Dentistry, Swami Devi Dyal Hospital and Dental College, Panchkula, Haryana, India.

Contact Corresponding Author At: sahrishtariqdario7[at]gmail[dot]com 